\title{
Moderate hyperoxia induces extracellular matrix remodeling by human fetal airway smooth muscle cells
}

\author{
Elizabeth R. Vogel ${ }^{1,2}$, Rodney D. Britt Jr ${ }^{2}$, Arij Faksh ${ }^{3}$, Ine Kuipers ${ }^{1}$, Hitesh Pandya ${ }^{4}$, Y.S. Prakash ${ }^{1,2}$, Richard J. Martin ${ }^{5}$ and \\ Christina M. Pabelick ${ }^{1,2}$
}

BACKGROUND: Premature infants are at increased risk for airway diseases, such as wheezing and asthma, because of early exposure to risk factors including hyperoxia. As in adult asthma, airway remodeling and increased extracellular matrix (ECM) deposition is involved.

METHODS: We assessed the impact of 24-72 $\mathrm{h}$ of moderate hyperoxia (50\%) on human fetal airway smooth muscle (fASM) ECM deposition through western blot, modified in-cell western, and zymography techniques.

RESULTS: Hyperoxia exposure significantly increased collagen I and collagen III deposition, increased pro- and cleaved matrix metalloproteinase 9 (MMP9) activity, and decreased endogenous MMP inhibitor, TIMP1, expression. Hyperoxiainduced change in caveolin-1 (CAV1) expression was assessed as a potential mechanism for the changes in ECM deposition. CAV1 expression was decreased following hyperoxia. Supplementation of CAV1 activity with caveolar scaffolding domain (CSD) peptide abrogated the hyperoxia-mediated ECM changes.

CONCLUSION: These results demonstrate that moderate hyperoxia enhances ECM deposition in developing airways by altering the balance between MMPs and their inhibitors (TIMPs), and by increasing collagen deposition. These effects are partly mediated by a hyperoxia-induced decrease in CAV1 expression. In conjunction with prior data demonstrating increased FASM proliferation with hyperoxia, these data further demonstrate that hyperoxia is an important instigator of remodeling in developing airways.

$\mathbf{P}$ ostnatal supplemental oxygen (hyperoxia) remains a significant risk factor for the development of pediatric and neonatal lung diseases. Premature infants are at particular risk because of their immature pulmonary systems and their disproportionate exposures to hyperoxia and/or respiratory support such as mechanical ventilation. While perinatal exposure to hyperoxia has been most commonly associated with alveolar disease such as bronchopulmonary dysplasia (BPD), there is increasing evidence that hyperoxia exposure has significant impact on the developing airway and may predispose to chronic airway diseases such as wheezing and asthma (1-4). Indeed, reactive airway diseases are the major long-term sequelae in infants who survive BPD, and they also affect infants who have no demonstrated evidence of BPD. These airway effects are often chronic, and may predispose to childhood, and even adult, asthma.

Airway remodeling, characterized by increased airway smooth muscle (ASM) mass and increased extracellular matrix (ECM) deposition in the airway wall, is a key feature of reactive airway diseases such as asthma (5-8). Prior studies by our group have demonstrated the proproliferative effects of moderate levels of hyperoxia (up to 50\%) in human fetal airway smooth muscle (fASM) cells, while higher levels of oxygen lead to increased apoptosis (9). While these changes in ASM proliferation are intriguing, the effects of hyperoxia on the broader aspects of airway remodeling, particularly ECM effects, have not been investigated in the developing airway. Of note, there is growing appreciation for the relationship between ASM and the ECM. Intriguingly, there is increasing evidence that ASM is an important modulator of the ECM, making this relationship a particularly interesting, though complex, one to study $(7,10-13)$.

The ECM is a dynamic structure that is composed of numerous components, including collagens, fibronectin, and proteoglycans. Breakdown of the ECM and/or changes to its composition can influence cell motility, proliferation, cellular signaling, and contractility (14). Increased ECM deposition may lead to stiffer more fibrotic airways. This can result in increased airway resistance, particularly in a still developing and more compliant neonatal lung. Of note, increased levels of collagen I and III precursors and mRNA have been found at autopsy in infants exposed to hyperoxia who developed BPD and airway disease $(1,15)$.

In addition, expression of key ECM modulators-matrix metalloproteinases (MMPs) - is elevated in infants with neonatal lung disease $(16,17)$. MMP2 and MMP9 are increased in

\footnotetext{
'Department of Anesthesiology, Mayo Clinic, Rochester, Minnesota; ${ }^{2}$ Physiology and Biomedical Engineering, Mayo Clinic, Rochester, Minnesota; ${ }^{3}$ Department of Obstetrics and Gynecology, Division of Maternal Fetal Medicine, Mayo Clinic, Rochester, Minnesota; ${ }^{4}$ Department of Pediatrics, University of Leicester, Leicester, UK; ${ }^{5}$ Department of Pediatrics, Division of Neonatology, Rainbow-Babies Children's Hospital, Case Western Reserve University, Cleveland, Ohio. Correspondence: Christina M. Pabelick (Pabelick.christina@mayo.edu)

Received 20 January 2016; accepted 19 August 2016; advance online publication 7 December 2016. doi:10.1038/pr.2016.218
} 
bronchoalveolar lavage (BAL) fluid samples of neonates with respiratory distress who develop BPD while increased collagen deposition has been noted in the pulmonary parenchyma of these infants on histologic assessment $(15,17,18)$. Hyperoxia exposure is one factor that has been shown to increase MMP expression (19). MMPs are zinc-dependent endopeptidases that are most commonly associated with their role in degradation of the extracellular matrix. The gelatinases (MMPs 2 and 9) have been specifically implicated in pulmonary disease as contributors to hyperoxia-induced lung disease and asthma $(17,20)$. Tissue inhibitors of metalloproteinases (TIMPs) are the primary regulators of MMPs; they are able to bind and inactivate them in a one to one ratio (21). The balance between MMPs and TIMPs is therefore an important one.

Caveolin-1 (CAV1) has emerged as an important regulator of remodeling in pulmonary diseases, including asthma. CAV1 is the primary structural element of caveolae, which are lipid-, cholesterol-, and sphingomyelin-rich membrane domains that serve as points of concentration for numerous cell-signaling components, including receptors, ion channels, and other key cell signaling components (22-24). Altered levels of CAV1 carry significant structural and functional implications (25-27).

CAV1 plays an important role in regulation of fibrosis and ECM deposition in the lung. A number of studies, including prior work from our group, have shown that CAV1 knock-out mice demonstrate increased airway hyper-reactivity, as well as increased airway remodeling with elevated levels of collagen and myofibroblasts (28-31). CAV1 and caveolae have also been shown to inhibit the secretion and activity of multiple MMPs, including MMPs 1, 2, and 9 (32). These multiple associations of CAV1 and caveolar signaling with remodeling and fibrosis make it an intriguing area of investigation for hyperoxia-induced injury in the developing airway.

In this study, we used fetal ASM cells (fASM) as an in vitro model of one important aspect of the developing airway, and we examined the effects of moderate hyperoxia exposure on fASM ECM deposition and on CAV1 expression. Alteration in CAV1 expression was assessed as a potential mechanism of hyperoxia-induced remodeling effects.

\section{METHODS}

\section{Cell Culture}

Deidentified and cultured human fASM cells were purchased from Novogenix (Los Angeles, CA) or provided by Dr. Pandya (University of Leicester, England). The Mayo Clinic IRB found these protocols and specimens IRB exempt. Leicester protocols were also approved by ethics committees at the University of Leicester. In brief, human tracheobronchial airway smooth muscle cells were enzymatically dissociated from 18 to $20 \mathrm{wk}$ gestational age (canalicular stage) lung tissue, as previously described (33). Isolated cells were not pooled together from multiple samples, and as such each sample or "n" represents cells isolated from an individual lung. Cells were cultured using standard conditions in 10\% fetal bovine serum (FBS), phenol red free Dulbecco's Modified Eagle Medium (DMEM)/F12 growth media. Prior to treatments, cells were growth arrested in $0.5 \%$ FBS media for $24 \mathrm{~h}$. Previously described smooth muscle markers (smooth muscle actin, calponin, and acetylcholine receptor) were used to confirm phenotype throughout the duration of experiments $(9,34)$. All cells were from passages $2-10$. Preliminary experiments performed in our lab demonstrated no phenotypic or behavioral changes between low and high passage cells within this range.

\section{Cellular Exposures}

fASM cells were exposed to $50 \%$ hyperoxia for $24-72 \mathrm{~h}$ following serum starvation in $0.5 \%$ FBS for a minimum of $24 \mathrm{~h}$. To investigate the role of CAV1 expression on the observed ECM changes, an analog of the CSD peptide was used. Native, nontransfected cells were pretreated with $1 \mu \mathrm{mol} / \mathrm{l} \mathrm{CSD}$ for $30 \mathrm{~min}$ prior to hyperoxia exposure.

\section{Western Blot}

Whole cell lysates or concentrated conditioned media were separated on SDS-PAGE gels (7, 10, or 4-15\% gradient gels, Criterion Gel System; Bio-Rad, Hercules, CA) via standard techniques. Proteins were transferred to nitrocellulose membranes using a Bio-Rad TransBlot Turbo system and blocked in Odyssey Blocking buffer (Li-Cor Biosciences, Lincoln, NE) prior to overnight incubation in a $1 \mu \mathrm{g} / \mathrm{ml}$ solution of primary antibody of interest. Primary antibodies included: caveolin-1, collagen I, collagen III (Abcam, Cambridge, MA), Cavin1, Cavin-3, fibronectin, matrix metalloproteinase 2 (MMP2), matrix metalloproteinase 9 (MMP9), tissue inhibitor of metalloproteinase 1 (TIMP1), and tissue inhibitor of metalloproteinase 2 (TIMP2) (Santa Cruz Biotechnology, Santa Cruz, CA). Secondary antibodies were conjugated to $800 \mathrm{CW}$ or $680 \mathrm{CW}$ IR dye (Li-Cor Biosciences) and imaging was performed using a Li-Cor OdysseyXL system prior to quantification via densitometry. Glyceraldehyde-3-phosphate dehydrogenase (GAPDH) expression was used to normalize cell lysate protein concentrations. Concentrated conditioned media was normalized to cell lysate protein concentrations as determined by Lowry protein assays.

\section{Zymography}

Standard protocols for gelatin zymography were used to measure MMP-2 and MMP-9 activity in concentrated media from fASM cells exposed to hyperoxia. Media was concentrated using Millipore centrifugal filter tubs (Beverly, MA). Samples were separated using 10\% sodium dodecyl sulfate polyacrylamide gel electrophoresis (SDSPAGE) gelatin zymography gels (Bio-Rad). Gels were developed using Bio-Rad renaturization and developing buffers according to the recommended protocols. Gels were stained in Coomassie blue, destained in a solution of $50 \%$ methanol, $40 \%$ DI water, and $10 \%$ acetic acid, and then quantified by densitometry using a Li-Cor Odyssey XL system. Activity was standardized to cell lysate protein concentration.

\section{Modified In-Cell Western}

Extracellular matrix deposition was measured using a modified incell western technique, as previously described $(34,35)$. Briefly, cells grown to $60 \%$ confluence in 96 -well plates were exposed to normoxia or $50 \%$ hyperoxia for $72 \mathrm{~h}$. Cells were then lysed with $0.016 \mathrm{~N}$ $\mathrm{NH}_{4} \mathrm{OH}$, rinsed with TBS, and blocked in Li-Cor Odyssey Blocking buffer prior to overnight incubation in the primary antibody of interest at a $10 \mu \mathrm{g} / \mathrm{ml}$ concentration. Primary antibodies included collagen I and collagen III (Abcam) and fibronectin (Santa Cruz). Plates were incubated in IR-conjugated secondary antibodies at a concentration of $5 \mu \mathrm{g} / \mathrm{ml}$ for $60 \mathrm{~min}$. Quantification was determined via densitometry after imaging with a Li-Cor OdysseyXL system. Blank wells without cells were treated with media and all primary and secondary antibodies to control for any background signal.

\section{siRNA Transfection}

Lipofectamine was used to transfect $60-70 \%$ confluent fASM cells with scrambled or caveolin-1 siRNA under serum-free, antibioticfree conditions. $20 \%$ FBS media was added, producing $10 \%$ FBS conditions for overnight incubation. Cells were then serum starved and exposed to $50 \%$ hyperoxia for $24 \mathrm{~h}$. Transfection efficiency was determined by assessing caveolin-1 expression by western blot.

\section{Statistical Analysis}

A minimum of four fASM samples, with two replicates per sample, were used for all experiments. A Sigma Plot software package (SYSTAT, San Jose, CA) was used for statistical analysis. Data were analyzed via Students $t$-test or one-way ANOVA, followed by Student-Newman-Keuls post hoc analysis. Statistical significance was set at $P<0.05$, with values reported as mean \pm SEM. 


\section{RESULTS}

\section{Hyperoxia Increases fASM Extracellular Matrix Production}

A modified in-cell western technique was used to assess the impact of moderate hyperoxia exposure on three ECM components: collagen I, collagen III, and fibronectin. Cells were exposed to $50 \%$ oxygen or $21 \%$ oxygen for $72 \mathrm{~h}$ prior to analysis. Hyperoxia exposure significantly increased collagen I and collagen III deposition, but did not impact fibronectin deposition $(P<0.05$, Figure 1$)$. Blank control wells did not demonstrate fluorescence.

\section{Hyperoxia Modulates MMP and TIMP Activity and Expression}

MMP2 and MMP9 are gelatinases involved in ECM remodeling that have been found to have increased expression in BAL samples from neonates with BPD and reactive airway disease $(18,19)$. In light of this clinical data, we used gelatin zymography to assess MMP2 and MMP9 activity in concentrated media from fASM cells exposed to hyperoxia vs. room air. Hyperoxia exposure significantly increased pro- and cleaved MMP9 activity, but it did not affect MMP2 activity (Figure 2).

To determine whether this change in MMP9 activity was due to increased expression of MMP9 vs. increased activation/loss of inhibition, western analysis of MMP2, MMP9, and MMP inhibitors TIMPs 1 and 2 (TIMPs) was performed on concentrated media from control vs. hyperoxia-exposed fASM cells. Western blot protein expression in concentrated media was normalized to the protein concentrations of the corresponding lysates. Expression of TIMP1 was significantly decreased with hyperoxia exposure, while expression of TIMP2, MMP2, and MMP9 was unchanged (Figure 3). It is likely that the decrease in TIMP1 expression contributes to the increase in MMP9 activity because TIMP1 is a major regulator of MMP9.

\section{Hyperoxia Impacts Caveolar Protein Levels}

Western blot analysis was used to assess the impact of hyperoxia on caveolar protein levels as a possible mechanism for the hyperoxia-induced changes in ECM proteins and ECM modulators. CAV1 expression was significantly decreased with hyperoxia $(P<0.05$, Figure 4$)$. Expression of cavin- 1 and cavin-3, constituent proteins involved in plasma membrane insertion and removal of CAV1, was unchanged.

\section{CAV1 Modulates ECM Remodeling in Hyperoxia-Exposed fASM}

To assess the role of decreased CAV1 expression on fASM ECM deposition, cells were transfected with CAV1 siRNA, and expression of ECM molecules in concentrated media was assessed via western blot. CAV1 siRNA resulted in increased collagen III in concentrated media in both room air and hyperoxic conditions. Collagen I was significantly increased with the combination of CAV1 siRNA transfection and hyperoxia exposure. These effects of the CAV1 siRNA were more pronounced with hyperoxia exposure $(P<0.05$, Figure 5$)$.

In an additional set of experiments, nontransfected cells were treated with caveolar scaffolding domain (CSD) (an ana$\log$ of the active portion of CAV1) prior to hyperoxia exposure to assess the effects of supplementation of CAV1 on ECM expression with hyperoxia exposure. CSD pretreatment abrogated the hyperoxia-induced increase in collagen I and III expression in concentrated media $(P<0.05$, Figure 6). There was no change in fibronectin expression.

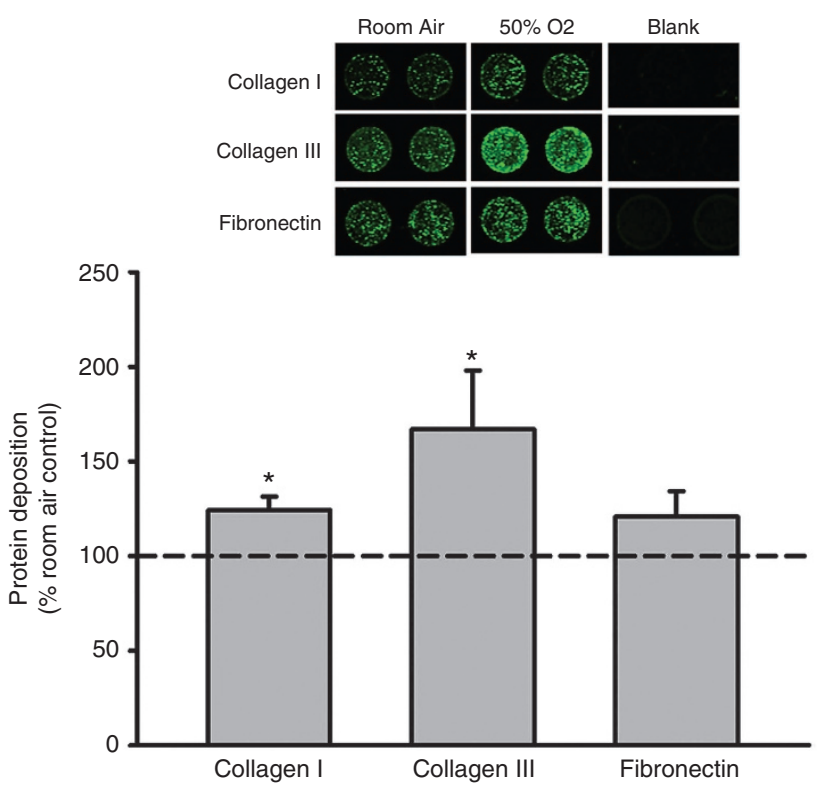

Figure 1. Moderate hyperoxia increases fetal airway smooth muscle (fASM) extracellular matrix deposition. Exposure of fASM cells to 50\% hyperoxia for $72 \mathrm{~h}$ significantly increased deposition of collagen I and collagen III. Fibronectin deposition was not affected. Values are means \pm SEM from $n$ of 4 samples. * indicates significant difference from $21 \% \mathrm{O}_{2}$ control with $P<0.05$.

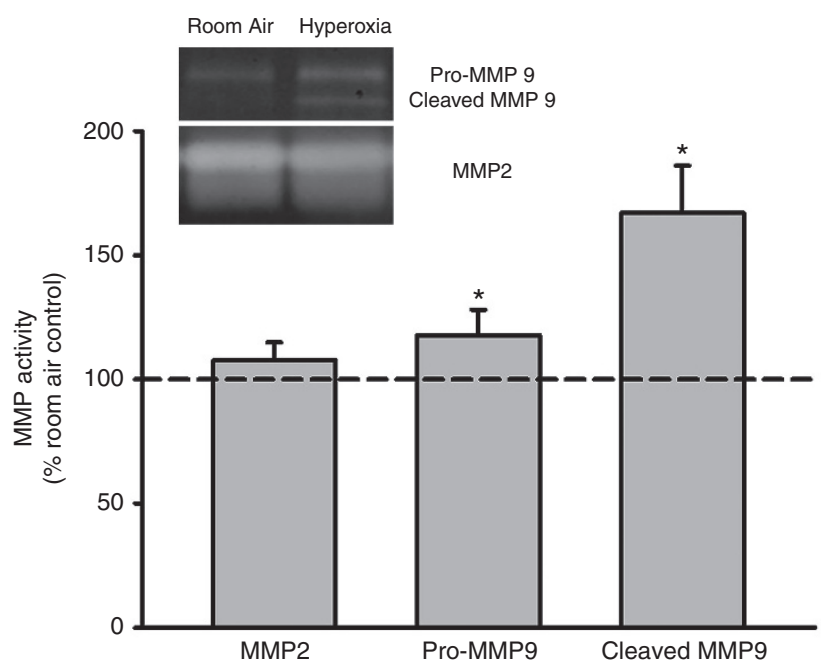

Figure 2. The effect of moderate hyperoxia on matrix metalloproteinase (MMP) activity. Fetal airway smooth muscle cells were exposed to 21 or $50 \%$ oxygen for $24 \mathrm{~h}$. Conditioned media was collected, concentrated, and analyzed via gelatinase zymograph for MMP9 and MMP2 activity. MMP activity in concentrated media was normalized to cellular lysate protein concentration. Both pro- and cleaved MMP9 activity were significantly increased with hyperoxia exposure. MMP2 activity was not changed. Values are means \pm SEM from $n$ of 4 samples. * indicates significant difference from $21 \% \mathrm{O}_{2}$ control with $P<0.05$. 
a

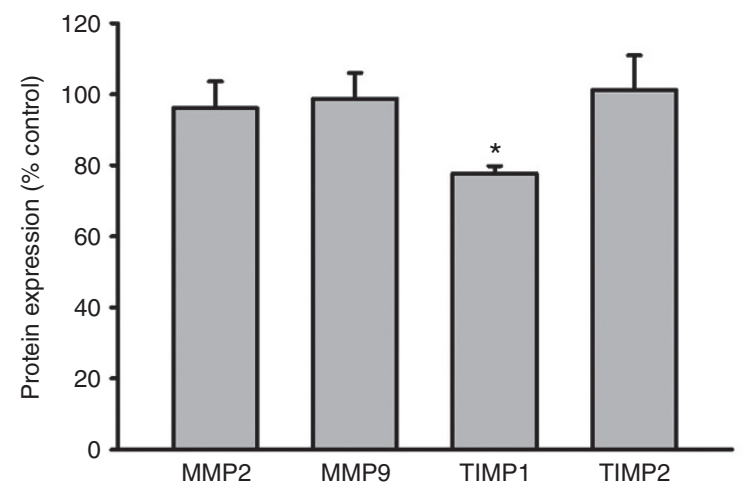

b

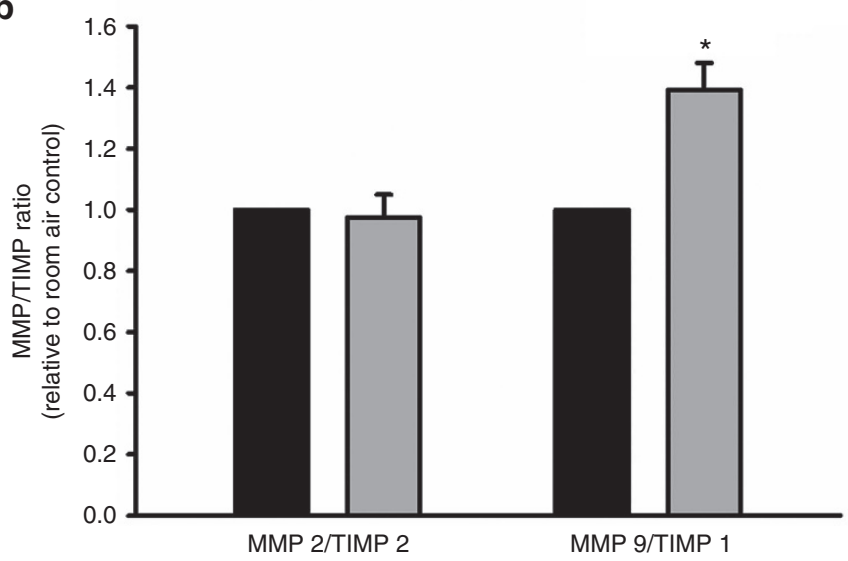

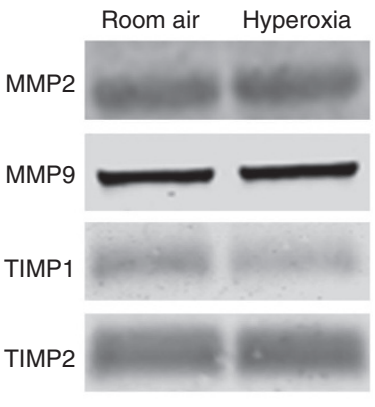

Figure 3. Impact of moderate hyperoxia on matrix metalloproteinase (MMP) and tissue inhibitors of metalloproteinase (TIMP) expression and ratios. fetal airway smooth muscle cells were exposed to 21 or $50 \%$ oxygen for $24 \mathrm{~h}$. Conditioned media was collected, concentrated, and analyzed via western blot for MMP and TIMP expression. Protein expression in the conditioned media was normalized to the protein concentration of the corresponding cellular lysate. (a) Hyperoxia significantly decreased TIMP1 expression. MMP2, MMP9, and TIMP2 expression was unaffected. (b) MMP9/TIMP1 ratio was significantly increased while MMP2/TIMP2 ratio was unaffected. Values are means \pm SEM from $n$ of 4 samples. Legend: Black $=21 \%$ oxygen, gray $=50 \%$ oxygen. ${ }^{*}$ indicates significant difference from $21 \% \mathrm{O}_{2}$ control with $P<0.05$.

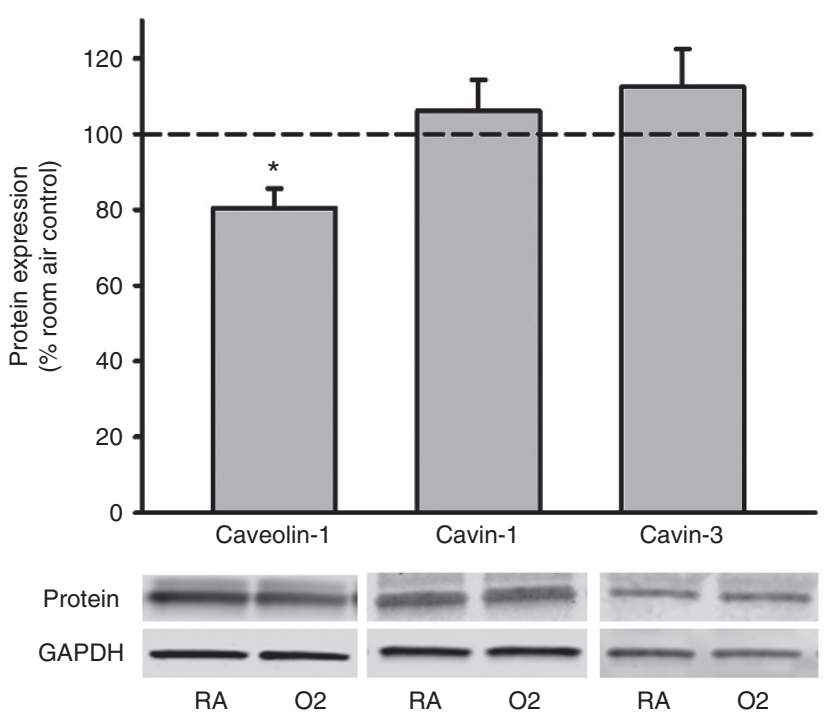

Figure 4. Effect of hyperoxia on caveolar protein expression in fetal airway smooth muscle cells. Cells were exposed to 21 or $50 \%$ oxygen for 24h. Hyperoxia exposure significantly decreased caveolin-1 expression but did not impact cavin-1 or cavin-3 expression. Values are means \pm SEM from $n$ of 4 samples. * indicates significant difference from $21 \% \mathrm{O}_{2}$ control with $P<0.05$.
To assess the impact of decreased CAV1 expression on MMP activity, we used gelatin zymography to assess MMP2 and MMP9 activity in concentrated media from fASM cells transfected with CAV1 siRNA. CAV1 siRNA transfection had no impact on MMP2 or MMP9 activity (Figure 7a). Similarly, we assessed MMP2, MMP9, TIMP1, and TIMP2 protein expression in concentrated media from transfected cells. There was no change in protein expression of any of these ECM modulators with CAV1 siRNA transfection (Figure 7b).

\section{DISCUSSION}

In this study, we examined the impact of hyperoxia exposure on expression and deposition of ECM molecules and mediators in primary human fASM cells. Exposure to hyperoxia resulted in a significant increase in collagen I and collagen III deposition in the context of increased pro- and cleaved MMP9 activity. TIMP1 activity was decreased, and this likely contributed to the observed increase in MMP9 activity. CAV1 expression was also significantly decreased with hyperoxia exposure; this implies a potential mechanism for the observed changes in ECM expression. Supplementation of CAV1 activity (using CSD peptide) ameliorated the hyperoxia-induced changes in collagen I deposition. Overall, these data demonstrate that 
moderate hyperoxia can induce remodeling in fASM cells and could contribute to airway remodeling in neonates exposed to hyperoxia.

Supplemental oxygen remains a common perinatal exposure. It has been well established that it is associated with the development of pulmonary diseases, including airway diseases such as wheezing and asthma. Clinical practice has been modified to minimize neonatal hyperoxia exposure and moderate levels $(<50 \%)$ of hyperoxia are now used to attempt to decrease the potential negative repercussions. However, even moderate hyperoxia causes abnormal redox stress in an immature and developing lung that is used to the relatively hypoxic intrauterine environment. We chose a moderate (50\%) level of hyperoxia because prior in vitro and in vivo murine studies have demonstrated increased ASM reactivity and remodeling behavior under similar conditions (3,36-39). Actual in vivo ASM oxygen exposure may be much lower since inspired oxygen is adjusted to target oxygen saturation in the low to mid 90's in most clinical settings. It is, however, possible that ASM in the premature airway is exposed to higher levels of oxygen than in a healthy airway due to epithelial breakdown and barrier dysfunction in the setting of prolonged hyperoxia exposure in the NICU postnatally. It remains important to better understand the response of the ASM to hyperoxia to help determine ways to prevent the deleterious effects of oxygen exposure in the developing lung. In this study, we demonstrated that moderate hyperoxia increases ECM deposition and that it also causes remodeling in fASM cells.

Airway remodeling and wall thickening is a primary structural change that occurs in reactive airway disease $(6,12,40)$. This wall thickening occurs through a combination of ASM proliferation and increased ECM deposition, resulting in stiffer, fibrotic airways. These changes can decrease airway caliber, which may lead to wheezing and obstructive airway pathophysiology $(6,12,14)$. We have previously demonstrated that hyperoxia increases proliferation of fetal ASM cells (9). Intriguingly, this effect was dose-dependent, with moderate hyperoxia (40-60\%) resulting in increased proliferation, while higher levels $(>60 \%)$ resulted in increased apoptosis. In addition to the proliferative effects of hyperoxia, we also previously demonstrated that high levels $(>70 \%)$ of oxygen exposure result in mitochondrial dysfunction and fission. In the current study, we have demonstrated that moderate hyperoxia also modulates ASM deposition of ECM molecules, increasing deposition of collagens I and III, and potentially contributing further to airway remodeling and airway dysfunction.

Prior studies demonstrate that increased ECM deposition in the developing lung occurs in the setting of BPD, respiratory distress syndrome (RDS), and hyperoxia exposure. However, the majority of these studies have focused on alveolar ECM deposition and BPD in the setting of high (>60\%) levels of oxygen. A study of pulmonary tissue from infants who died of RDS or BPD found increased collagen I and III precursor proteins and mRNA throughout the lungs of these infants (15). A recent publication focused on the impact of neonatal hyperoxia

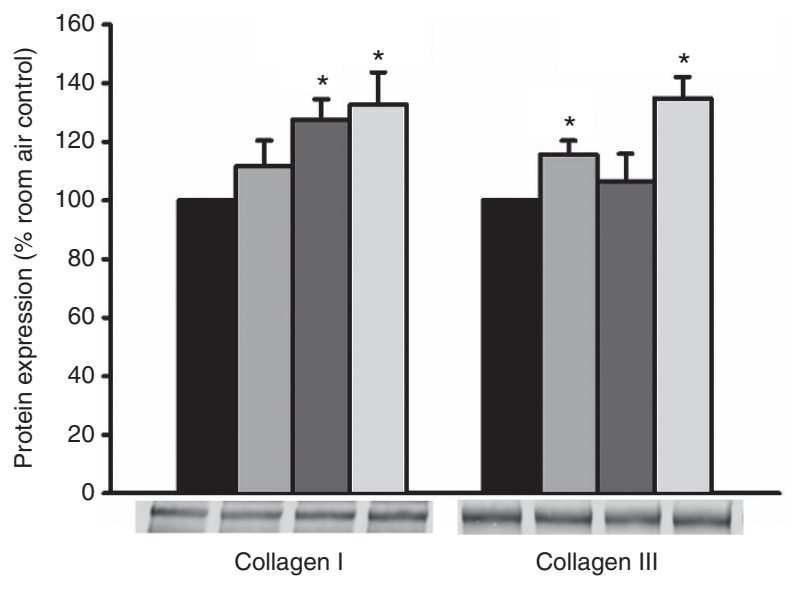

Figure 5. CAV1 knockdown increases extracellular matrix (ECM) expression in fetal airway smooth muscle (fASM) cells. fASM cells were transfected with CAV1 or scrambled siRNA prior to exposure to 21 or $50 \%$ oxygen. Knockdown of CAV1 increased collagen III expression in 21\% oxygen. Both collagen I and III were significantly increased following CAV1 transfection and 50\% oxygen exposure. Transfection with scrambled siRNA did not result in significant ECM change from control (data not shown). Protein expression in concentrated media was normalized to cellular lysate protein concentration. Values are presented as mean $\pm \mathrm{SEM}$. Legend: Black $=21 \%$ oxygen, light gray $=21 \%$ oxygen transfected with CAV 1 siRNA, dark gray $=50 \%$ oxygen, cream $=50 \%$ oxygen transfected with CAV1 siRNA. * indicates significant difference from $21 \%$ control.

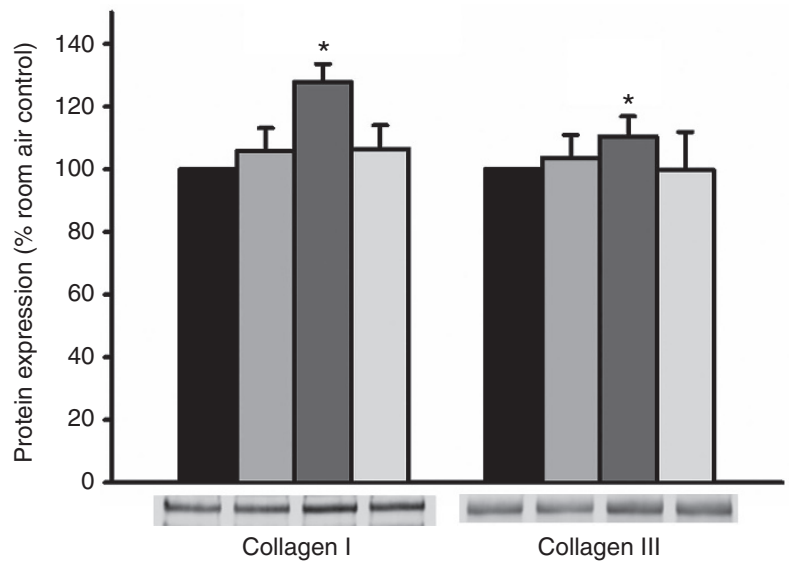

Figure 6. Pretreatment with caveolar scaffolding domain (CSD) peptide abrogates hyperoxia-induced extracellular matrix deposition. Cells were treated with vehicle or CSD peptide for $30 \mathrm{~min}$ prior to exposure to 21 or $50 \%$ oxygen for $24 \mathrm{~h}$. Concentrated conditioned media was analyzed for protein expression. Protein expression in concentrated media was normalized to cellular lysate protein concentration. Pretreatment with CSD peptide prevented hyperoxia-induced increases in collagen I and collagen III expression. Values are means \pm SEM from $n$ of 4 samples. Legend: Black $=21 \%$ oxygen, light gray $=21 \%$ oxygen plus CSD treatment, dark gray $=$ $50 \%$ oxygen, cream $=50 \%$ oxygen plus CSD treatment. ${ }^{*}$ indicates significant difference from $21 \% \mathrm{O}_{2}$ control with $P<0.05$.

on the murine airway; it found increased collagen deposition at $21 \mathrm{~d}$ of age in the airways with both 40 and $70 \%$ oxygen exposure (39). There is also evidence that early exposure to hyperoxia can have a long-term impact on airway collagen content. Mice exposed to $65 \%$ oxygen at birth that subsequently demonstrated growth restriction have been found to have increased airway collagen deposition in adulthood (56 d 
a

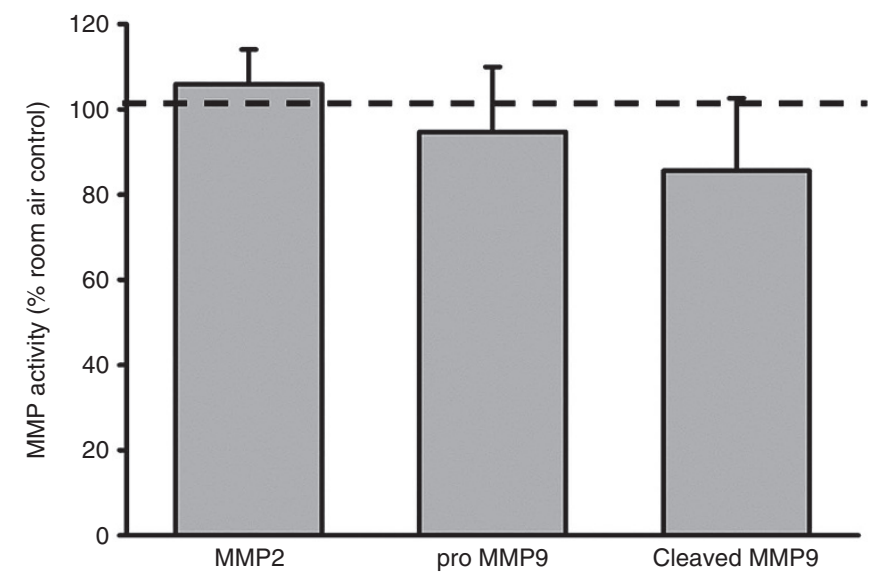

b

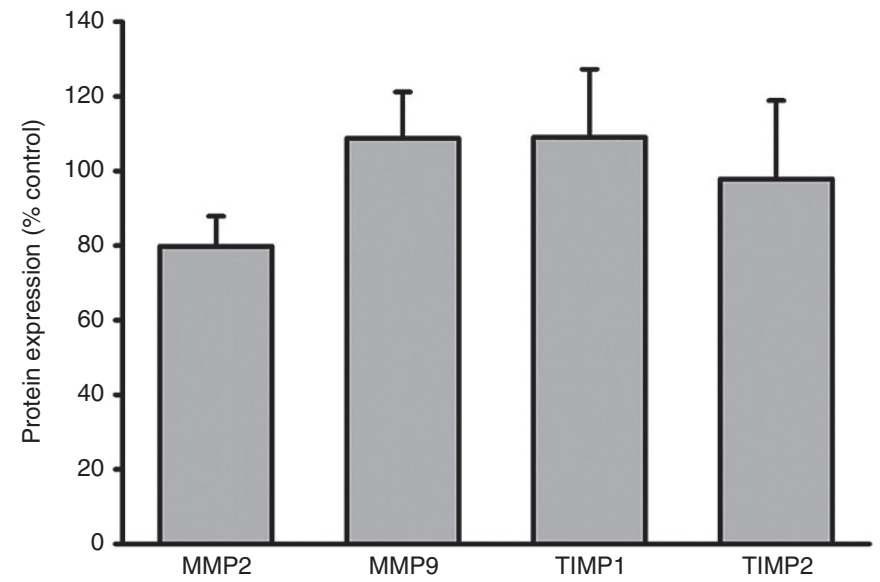

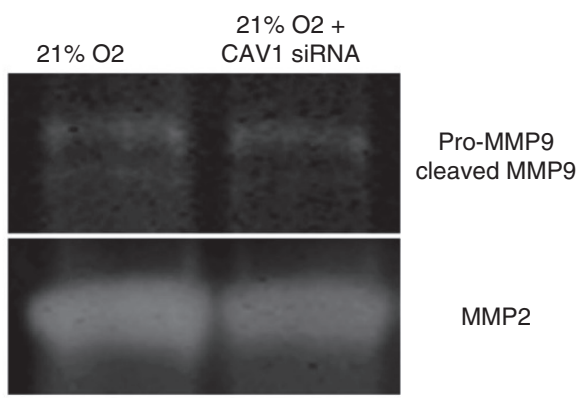

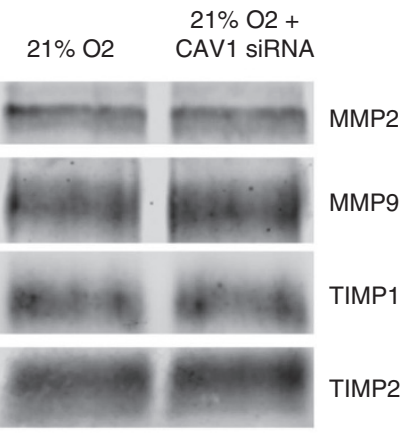

Figure 7. Impact of CAV1 knockdown on matrix metalloproteinase (MMP) activity and MMP and tissue inhibitors of metalloproteinase (TIMP) expression. Fetal airway smooth muscle cells were exposed to $21 \%$ with or without CAV1 siRNA transfection. (a) Conditioned media was collected, concentrated, and analyzed via gelatinase zymograph for MMP2 and MMP9 activity. There was no effect of CAV1 siRNA transfection on MMP activity. (b) Conditioned media was collected, concentrated and analyzed via western blot for MMP and TIMP expression. Protein expression in the conditioned media was normalized to the protein concentration of the corresponding cellular lysate. There was no effect of CAV1 siRNA transfection on MMP or TIMP protein expression in concentrated media. Values are means \pm SEM from $n$ of 4 samples. ${ }^{*}$ indicates significant difference from $21 \% \mathrm{O}_{2}$ control with $P<0.05$.

old) $(37,38)$. Our data suggest that ASM deposition of collagen in response to hyperoxia may be an important contributor to these changes.

Similarly, altered levels of MMPs have been noted in BAL specimens from preterm infants; the highest levels have been found in those who develop BPD and pulmonary disease $(17,18,20,41)$. MMPs are the primary regulators of the ECM; they breakdown the matrix to allow for new matrix formation, cell migration, and release of growth factors and other signaling molecules contained in the ECM $(42,43)$. We found increased activity of both the pro- and cleaved forms of MMP9 with moderate hyperoxia exposure. Interestingly, MMP9 is commonly cited as elevated in BALs from neonates with pulmonary disease, and it has been implicated as a key mediator of injury in hyperoxia-induced lung injury models of BPD. A murine model of BPD using exposure to high levels (85\%) oxygen demonstrated increased levels of MMP9 as well as increased whole lung collagen expression. Knockout of MMP9 ameliorated these effects (19). Increased levels of MMP9 and decreased TIMP1 have also been found in baboon models of hyperoxia-induced alveolar disease (44). Our findings were similar: The increased MMP9 activity we found is likely due to decreased TIMP1 expression with hyperoxia exposure because TIMP1 is a primary inhibitor of MMP9 activity.

Caveolae are important sites for cellular regulation, signal integration, and cross-talk of signaling pathways because of the number of ion channels and receptors they contain $(22,24)$. CAV1 is a particularly important cellular mediator; it is involved in numerous pathways, including cellular signaling, fibrosis, and proliferation. Decreased levels of CAV1 are associated with increased fibrosis and ECM deposition $(22,24,45)$. In light of these prior studies, we evaluated the effect of moderate hyperoxia on CAV1 expression in fASM cells as a potential mechanism for the increased ECM deposition that is observed with hyperoxia exposure. CAV1 levels were decreased with hyperoxia, while cavin levels were unchanged. This finding demonstrates the importance of CAV1 on airway structure and function.

More interesting is the fact that replacement of CAV1 activity through treatment with CSD peptide reversed the observed 


\section{Articles | vogelet al.}

changes in ECM expression that occurred in response to hyperoxia exposure. Similarly, CAV1 knockdown via siRNA transfection increased ECM expression in concentrated media. These changes in ECM expression are similar to prior studies that have found increased collagen expression with CAV1 knockdown. However, to our knowledge, there is no prior evidence that implicates hyperoxia as a mediator of CAV1 expression. Of note, these CAV1-mediated changes in ECM expression do not appear to be mediated through changes activity or expression of MMP2, MMP9, TIMP1, or TIMP2. It is more likely that the ECM changes result from loss of CAV1 suppression of other proliferative and fibrotic pathways, such as TGF- $\beta$. Modulation of CAV1 expression may represent a newly identified mechanism through which hyperoxia negatively impacts the developing airway. In light of the availability of the CAV1 analog, CSD peptide, this may represent a possible point of intervention to ameliorate the deleterious effects of oxygen on the developing airway. Further study is needed to better understand the impact of hyperoxia on CAV1 expression in the airway, and to explore the potential therapeutic implications.

The use of fetal ASM cells was both a limitation and advantage in this study. Most previous studies of hyperoxia exposure in the airway have been performed in adult cell lines, mature adult airway samples, or in animal models. The ability to use developing human ASM cells provides a unique opportunity to better understand the biology of the developing human airway. Limitations of our data include the fact that these are fetal ASM cells which may somewhat limit the generalizability of this data. All cells were from the canalicular stage, at $18-20 \mathrm{wk}$ gestational age, which is the phase of lung development where terminal airway branching takes places and preliminary gas exchange becomes possible. Premature infants born during the late canalicular stage are at the earliest stage of viability making this a relevant clinical time point for study. We were blinded as to exact gestational age of each sample. While there may be some variability depending on exact gestational age, the overall results were the same across the samples.

In summary, our findings demonstrate that moderate hyperoxia induces ECM remodeling behavior in developing fASM. Hyperoxia exposure increased collagen I and III deposition and MMP9 activity. These data demonstrate that moderate hyperoxia can induce airway remodeling that may then contribute to the development of airway thickening, thereby contributing to development of airway diseases such as wheezing and asthma. CAV1 expression was significantly decreased, and supplementation of CAV1 activity with the CSD peptide abrogated the hyperoxia-induced effects on collagen expression. In light of these data, CAV1 signaling likely plays a role in mediating the hyperoxia-induced ECM effects. CSD peptide may open a new avenue for therapeutic intervention in neonates exposed to supplemental oxygen.

\section{STATEMENT OF FINANCIAL SUPPORT}

This study was supported by the National Heart Lung and Blood Institute of the National Institutes of Health (T32 HL105355 (E.R.V.); F32 HL123075 (R.D.B.), and R01s HL088029, HL056470 (Y.S.P.), a grant through the Children's Research Center, Mayo Clinic, and the Mayo Clinic Department of Anesthesiology.
Disclosure: The authors report no conflicts of interest or disclosures.

\section{REFERENCES}

1. Bhandari V. Hyperoxia-derived lung damage in preterm infants. Semin Fetal Neonatal Med 2010;15:223-9.

2. Denis D, Fayon MJ, Berger P, et al. Prolonged moderate hyperoxia induces hyperresponsiveness and airway inflammation in newborn rats. Pediatr Res 2001;50:515-9.

3. Jobe AH, Kallapur SG. Long term consequences of oxygen therapy in the neonatal period. Semin Fetal Neonatal Med 2010;15:230-5.

4. Martin RJ, Prakash YS, Hibbs AM. Why do former preterm infants wheeze? J Pediatr 2013;162:443-4.

5. Araujo BB, Dolhnikoff M, Silva LF, et al. Extracellular matrix components and regulators in the airway smooth muscle in asthma. Eur Respir J 2008;32:61-9.

6. Dekkers BG, Maarsingh H, Meurs H, Gosens R. Airway structural components drive airway smooth muscle remodeling in asthma. Proc Am Thorac Soc 2009;6:683-92.

7. Hirota N, Martin JG. Mechanisms of airway remodeling. Chest 2013;144:1026-32.

8. Manuyakorn W, Howarth PH, Holgate ST. Airway remodelling in asthma and novel therapy. Asian Pac J Allergy Immunol 2013;31:3-10.

9. Hartman WR, Smelter DF, Sathish V, et al. Oxygen dose responsiveness of human fetal airway smooth muscle cells. Am J Physiol Lung Cell Mol Physiol 2012;303:L711-9.

10. Prakash YS. Airway smooth muscle in airway reactivity and remodeling: what have we learned? Am J Physiol Lung Cell Mol Physiol 2013;305:L91233.

11. Salerno FG, Barbaro MP, Toungoussova O, Carpagnano E, Guido P, Spanevello A. The extracellular matrix of the lung and airway responsiveness in asthma. Monaldi Arch Chest Dis 2009;71:27-30.

12. Siddiqui S, Martin JG. Structural aspects of airway remodeling in asthma. Curr Allergy Asthma Rep 2008;8:540-7.

13. Gosens R, Grainge C. Bronchoconstriction and airway biology: potential impact and therapeutic opportunities. Chest 2015;147:798-803.

14. Parameswaran K, Willems-Widyastuti A, Alagappan VK, Radford K, Kranenburg AR, Sharma HS. Role of extracellular matrix and its regulators in human airway smooth muscle biology. Cell Biochem Biophys 2006;44:139-46.

15. Kaarteenaho-Wiik R, Pääkkö P, Herva R, Risteli J, Soini Y. Type I and III collagen protein precursors and mRNA in the developing human lung. J Pathol 2004;203:567-74.

16. Schock BC, Sweet DG, Ennis M, Warner JA, Young IS, Halliday HL. Oxidative stress and increased type-IV collagenase levels in bronchoalveolar lavage fluid from newborn babies. Pediatr Res 2001;50:29-33.

17. Sweet DG, McMahon KJ, Curley AE, O’Connor CM, Halliday HL. Type I collagenases in bronchoalveolar lavage fluid from preterm babies at risk of developing chronic lung disease. Arch Dis Child Fetal Neonatal Ed 2001;84:F168-71.

18. Cederqvist $\mathrm{K}$, Sorsa T, Tervahartiala T, et al. Matrix metalloproteinases-2, -8 , and -9 and TIMP-2 in tracheal aspirates from preterm infants with respiratory distress. Pediatrics 2001;108:686-92.

19. Chetty A, Cao GJ, Severgnini M, Simon A, Warburton R, Nielsen HC. Role of matrix metalloprotease-9 in hyperoxic injury in developing lung. Am J Physiol Lung Cell Mol Physiol 2008;295:L584-92.

20. Sweet DG, Curley AE, Chesshyre E, et al. The role of matrix metalloproteinases -9 and -2 in development of neonatal chronic lung disease. Acta Paediatr 2004;93:791-6.

21. Visse R, Nagase H. Matrix metalloproteinases and tissue inhibitors of metalloproteinases: structure, function, and biochemistry. Circ Res 2003;92:827-39.

22. Gosens R, Mutawe M, Martin S, et al. Caveolae and caveolins in the respiratory system. Curr Mol Med 2008;8:741-53.

23. Gosens R, Stelmack GL, Dueck G, et al. Role of caveolin-1 in p42/p44 MAP kinase activation and proliferation of human airway smooth muscle. Am J Physiol Lung Cell Mol Physiol 2006;291:L523-34.

24. Thompson MA, Prakash YS, Pabelick CM. The role of caveolae in the pathophysiology of lung diseases. Expert Rev Respir Med 2014;8:111-22. 
25. Gosens R, Stelmack GL, Dueck G, et al. Caveolae facilitate muscarinic receptor-mediated intracellular $\mathrm{Ca} 2+$ mobilization and contraction in airway smooth muscle. Am J Physiol Lung Cell Mol Physiol 2007;293: L1406-18.

26. Maniatis NA, Chernaya O, Shinin V, Minshall RD. Caveolins and lung function. Adv Exp Med Biol 2012;729:157-79.

27. Sathish V, Yang B, Meuchel LW, et al. Caveolin-1 and force regulation in porcine airway smooth muscle. Am J Physiol Lung Cell Mol Physiol 2011;300:L920-9.

28. Aravamudan B, VanOosten SK, Meuchel LW, et al. Caveolin-1 knockout mice exhibit airway hyperreactivity. Am J Physiol Lung Cell Mol Physiol 2012;303:L669-81.

29. Le Saux CJ, Teeters K, Miyasato SK, et al. Down-regulation of caveolin-1, an inhibitor of transforming growth factor-beta signaling, in acute allergen-induced airway remodeling. J Biol Chem 2008;283:5760-8.

30. Razani B, Engelman JA, Wang XB, et al. Caveolin-1 null mice are viable but show evidence of hyperproliferative and vascular abnormalities. J Biol Chem 2001;276:38121-38.

31. Razani B, Woodman SE, Lisanti MP. Caveolae: from cell biology to animal physiology. Pharmacol Rev 2002;54:431-67.

32. Williams TM, Medina F, Badano I, et al. Caveolin-1 gene disruption promotes mammary tumorigenesis and dramatically enhances lung metastasis in vivo. Role of Cav-1 in cell invasiveness and matrix metalloproteinase (MMP-2/9) secretion. J Biol Chem 2004;279:51630-46.

33. Pandya HC, Snetkov VA, Twort CH, Ward JP, Hirst SJ. Oxygen regulates mitogen-stimulated proliferation of fetal human airway smooth muscle cells. Am J Physiol Lung Cell Mol Physiol 2002;283:L1220-30.

34. Britt RD Jr, Faksh A, Vogel ER, et al. Vitamin D attenuates cytokineinduced remodeling in human fetal airway smooth muscle cells. J Cell Physiol 2015;230:1189-98.
35. Vogel ER, VanOosten SK, Holman MA, et al. Cigarette smoke enhances proliferation and extracellular matrix deposition by human fetal airway smooth muscle. Am J Physiol Lung Cell Mol Physiol 2014;307:L978-86.

36. Hershenson MB, Aghili S, Punjabi N, et al. Hyperoxia-induced airway hyperresponsiveness and remodeling in immature rats. Am J Physiol 1992;262(3 Pt 1):L263-9.

37. O’Reilly M, Hansbro PM, Horvat JC, Beckett EL, Harding R, Sozo F. Bronchiolar remodeling in adult mice following neonatal exposure to hyperoxia: relation to growth. Anat Rec (Hoboken) 2014;297:758-69.

38. O’Reilly M, Harding R, Sozo F. Altered small airways in aged mice following neonatal exposure to hyperoxic gas. Neonatology 2014;105:39-45.

39. Wang H, Jafri A, Martin RJ, et al. Severity of neonatal hyperoxia determines structural and functional changes in developing mouse airway. Am J Physiol Lung Cell Mol Physiol 2014;307:L295-301.

40. Kwinta P, Pietrzyk JJ. Preterm birth and respiratory disease in later life. Expert Rev Respir Med 2010;4:593-604.

41. Harijith A, Choo-Wing R, Cataltepe S, et al. A role for matrix metalloproteinase 9 in IFN $\gamma$-mediated injury in developing lungs: relevance to bronchopulmonary dysplasia. Am J Respir Cell Mol Biol 2011;44:621-30.

42. Dancer RC, Wood AM, Thickett DR. Metalloproteinases in idiopathic pulmonary fibrosis. Eur Respir J 2011;38:1461-7.

43. Davey A, McAuley DF, O'Kane CM. Matrix metalloproteinases in acute lung injury: mediators of injury and drivers of repair. Eur Respir J 2011;38:959-70.

44. Tambunting F, Beharry KD, Hartleroad J, Waltzman J, Stavitsky Y, Modanlou HD. Increased lung matrix metalloproteinase-9 levels in extremely premature baboons with bronchopulmonary dysplasia. Pediatr Pulmonol 2005;39:5-14.

45. Tourkina E, Richard M, Gööz P, et al. Antifibrotic properties of caveolin-1 scaffolding domain in vitro and in vivo. Am J Physiol Lung Cell Mol Physiol 2008;294:L843-61. 